\title{
OBSERVATIONS BY THE HAMBURG SRS-EXPEDITION TO PERTH
}

\author{
ERIK HØG \\ Hamburger Sternwarte, F.R. Germany \\ and \\ I. NIK OLOFF \\ Perth Observatory, Western Australia
}

\begin{abstract}
A total of 110000 observations of 26000 stars was obtained between 1967 and 1972 . The internal mean errors are 0.17 and 0.27 in $\Delta \alpha \cos \delta$ and $\Delta \delta$, respectively, both multiplied by the factor $(\cos z)^{-0.5}$ at the zenith distance $z$. The preliminary evaluation also shows that the internal systematic errors, judged from the clamp corrections in $5^{\circ}$ zones in declination, are less than $\pm 0 . .^{\prime \prime} 02$ and \pm 0 " 15 , respectively. The systematic differences in right ascension from the FK4 are given.
\end{abstract}

\section{Introduction and Conclusions}

The Hamburg expedition conducted by J. von der Heide enjoyed the hospitality of, and a close collaboration with the Perth Observatory for many years. The observations of the SRS program were started in November 1967. After the departure of the eight German observers in December 1971 I. Nikoloff continued the observing program until August 1972 when about 110000 observations of R.A. and Decl.had been obtained during 580 nights.

The observations encompass 24803 observations of FK4 stars with $\delta<+38^{\circ}$, at least 4 observations of all stars with $\delta<-40^{\circ}$ and at least 3 observations for $80 \%$ of all program stars in the SRS catalogue. About $8 \%$ of the catalogue stars have only one observation and a few hundred have not been observed at all. Every observation of a star gave a magnitude $m_{v}$ with a mean error of $0^{m} \cdot 09$. In addition 560 observations of major and minor planets were obtained.

The photoelectric Repsold Meridian Circle, which is still in operation at Perth on a program of FK4 Supp stars, has been described by Høg (1972) and a preliminary analysis of 150 nights of observations has been given.

The following contains a more detailed analysis of the observations of the FK4 stars in right ascension and finally the difference (Cat. - FK4) for $\Delta \alpha_{\delta} \cos \delta$. Further checks on the internal and external accuracy are still being carried out, and in this sense the present analysis is only preliminary. As far as yet the following conclusions are justified:

(1) The clamp corrections for $5^{\circ}$ zones are about \pm 0 " 010 .

(2) The internal mean error of one observation of a star is 0.18 and the asymptotic mean error is less than 0.030 .

(3) The values of $\Delta \alpha_{\delta} \cos \delta$ in Figure 3 for $5^{\circ}$ zones have internal systematic errors less than \pm 0.020 and perhaps equal to only \pm 0.010 . 
(4) A photoelectric multislit micrometer improves not only the accidental errors but also the systematic errors, probably by diminishing the thermal and other influences from the observer on the whole instrument.

Thus, the performance of the photoelectric Repsold meridian circle is even better than the first 150 nights indicated. The high systematic accuracy has not been known before, and it should encourage the modernization of conventional meridian circles with new micrometers, circles and pivots, since a lot can be gained without introducing new types of meridian circles (Høg, 1973).

The accuracy of the observations in Decl. is better than of visual observations, the mean error being 0.27 and the systematic errors less than 0 ". 15 . The lesser accuracy in Decl. than in R.A. is caused by the imperfect circles and by our incomplete knowledge of the division errors.

\section{Right Ascensions of FK4 Stars}

The average observing night lasted $9 \mathrm{~h}$ and contained 4 observations of nadir and marks, 160 program stars and 43 FK4 stars. The FK4 stars were distributed equally during the night over an interval of $7^{\mathrm{h}}$ of R.A. and over the zenith distances $\pm 70^{\circ}$. The observations in R.A. of the night were corrected for the errors of collimation and drifts in inclination and azimuth by means of the observations of nadir and marks. The FK4 stars therefore served only to determine two constant parameters for a night, i.e. the rotation around two axes: azimuth and clock correction. The mean errors for the two parameters were 0 ."10 and 0 ".06, respectively, for a night.

The observations were designed to be relative to the FK4, but since they are linked so firmly to a large area of the sky every night, they can reveal certain systematic errors of the FK4. Attempts to observe the same stars in upper and lower culmination in one night were not successful due to the low latitude; neither were the nights linked together by assuming a constant direction to the marks from night to night as is usual in absolute observations.

A systematic correction of unknown origin to the collimation of about 0.2 , and constant over a few years, was introduced in order to remove part of the clamp differences. The resulting clamp corrections, i.e. half of the difference, is shown in Figure 1c, as derived from 24803 observations of FK4 stars. The pivot corrections derived from direct measurement on the pivots and shown in Figure la were not applied. Had they been real they would have caused the clamp corrections shown as ' $\frac{1}{2}(E-W)$ pivots' in Figure 1b. Obviously, however, the observed clamp corrections are smaller and show no similarity to the plot above. This proves that the pivots are extremely good with irregularities generally less than $0.05 \mu \mathrm{m}$. Actually the pivots are elliptical with an ellipticity about $0.15 \mu \mathrm{m}$ for both pivots. The axes of these ellipses are nearly parallel; whether by accident or by virtue of the manufacturer (Heidenreich und Harbeck, 1964) we do not know. Therefore, an ellipticity effect of the form $\sin (2 z+\psi)$ does not appear in R.A.

For comparison the pivot and clamp corrections for the Washington six-inch 


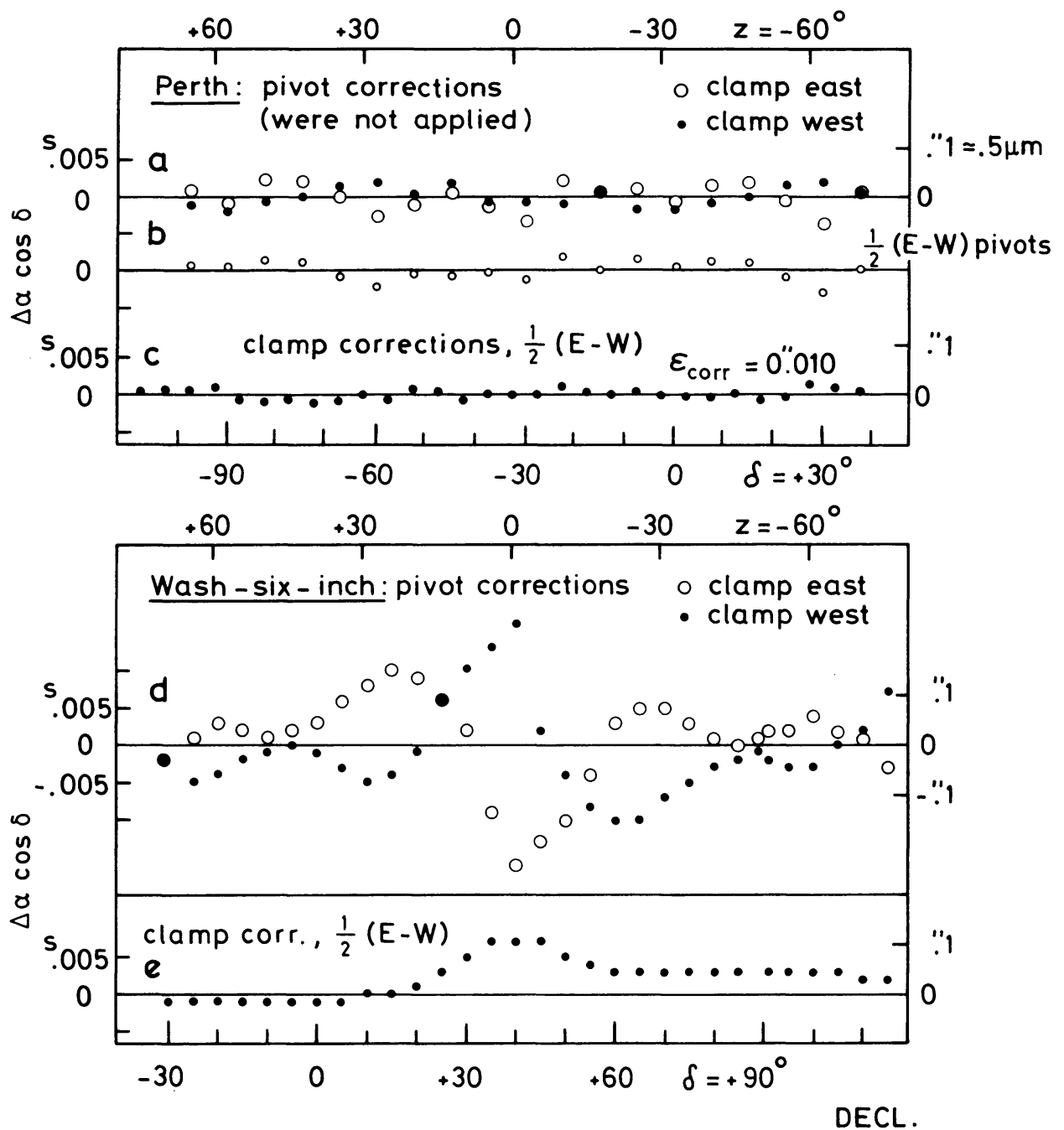

Fig. 1. Pivot corrections and clamp corrections for the meridian circles in Perth and Washington.

(Adams and Scott, 1968) are shown in Figures 1d and le. The rapid variation of the pivot corrections near zenith has apparently not been measured quite correctly so that larger clamp corrections remain in that region. The values in Figure le are smoothed between the $5^{\circ}$ zones, and their scatter therefore cannot be compared with the unsmoothed values in Figure 1c.

The residual scatter in Figure 1c is most simply expressed as $\varepsilon_{\text {corr }}=0$ ". $010=0.0007$ for a $5^{\circ}$ zone. Since each point is derived from about 400 observations in each clamp position we, therefore, have $\varepsilon_{400}=0.014$, where $\varepsilon_{n}$ is the mean error for the mean value of $n$ observations. This error must be due to pivot irregularities, image motion, etc.; the mean error $\varepsilon_{1}=0^{\prime \prime} 18$ for one observation contributing $0.18 / 400^{0.5}=0.009$. 
The internal mean errors for one observation are shown in Figure 2. The mean errors in R.A. from the 24603 observations within the $\pm 3 \sigma$ limits do not differ significantly whether observations from both clamp positions are taken together or whether they are treated separately, see Figures $2 b$ and $2 a-$ in agreement with the minute clamp corrections (which are not applied). The mean error increases less with the zenith distance than predicted by the theoretical law $(\cos z)^{-0.5}$ and may be represented by the value $\varepsilon_{1}=0$ " $18=0.012$ at all zenith distances $z \leqslant 50^{\circ}$.

Figures $2 \mathrm{c}$ and $2 \mathrm{~d}$ show the mean errors for Decl. which have a dependence on zenith distance nearly as predicted by the theoretical law. The values in Figure $2 \mathrm{~d}$ where both circles have been used for each star are much influenced by our incomplete knowledge of the division errors while Figure $2 \mathrm{c}$ is less dependent on this effect.

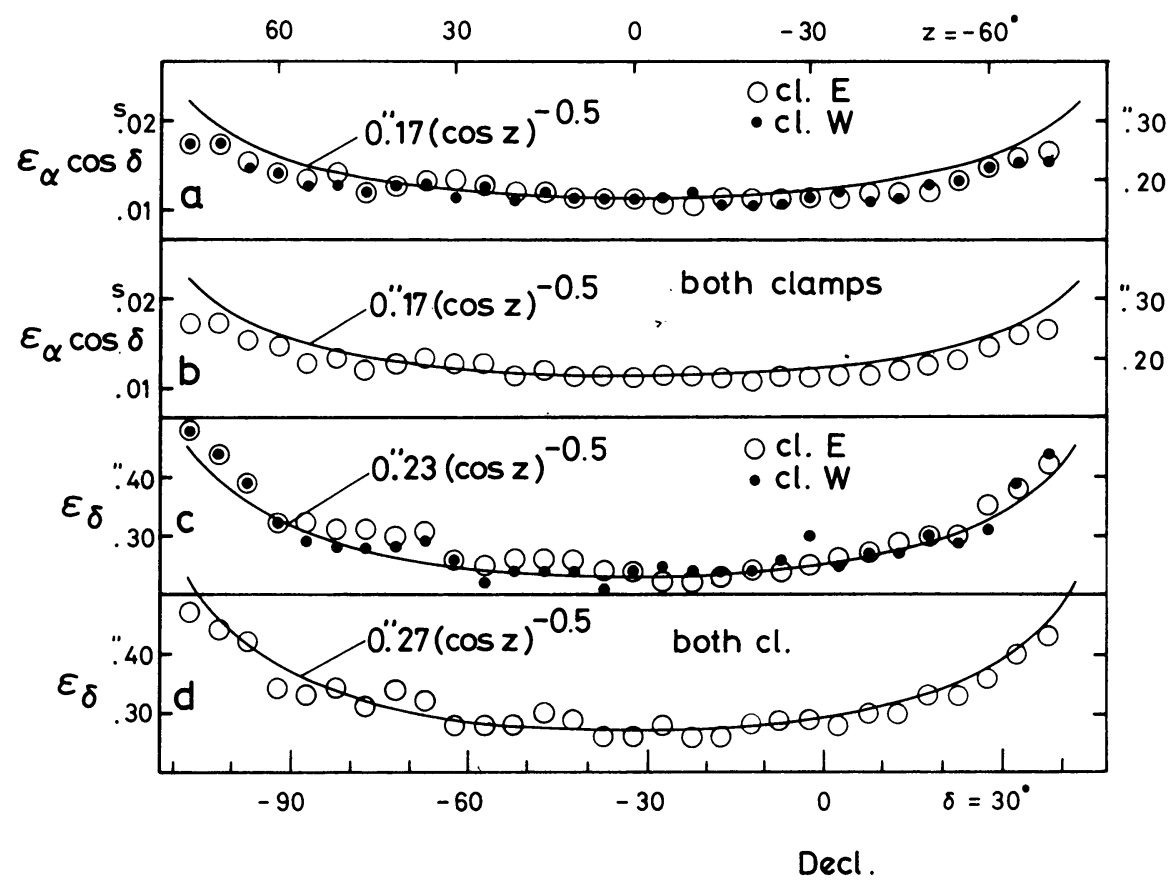

Fig. 2. Internal mean errors for one observation as function of zenith distance based on 24803 observations of FK4 stars. The mean errors are given for R.A. and for Decl. and for each of these separated in clamp east, clamp west and both clamp positions together.

The statistical error distribution* shall be given since it is a measure for the reliability of a catalogue. In this connection we mention the study by Dejaiffe (1973). The distribution of deviations of the single observations of R.A. from the mean values were found to differ significantly from a Gaussian in the wings (but probably less so

* The present values for the error distribution differ somewhat from the values given in the preprint distributed at the IAU Symposium. Also the mean errors at large zenith distances are slightly changed both due to the correction of two errors in the computer program. 
than other meridian observations). The percentages of deviations outside the limits $\pm n \sigma$, where $\sigma=0.18(\cos z)^{-0.5}$ and $n=1,2,3,4,6$ and 9, were $27.9(31.8), 4.5(4.6)$, $0.81(0.27), 0.28(0.006), 0.13(0.000)$, and $0.06(0.000)$, respectively. Values for a Gaussian are given in brackets. The percentages were derived from 24803 FK 4 observations, and the $0.81 \%$ of the observations outside $\pm 3 \sigma$ were rejected. Out of 85088 observations of program stars $0.74 \%$ deviations are outside $\pm 3 \sigma$. It is statistically important for small numbers of observations $n$ of a star to decrease the width of the limits by the factor $(1-(1 / n))^{-0.5}$, and this has be done throughout.

Returning to $\varepsilon_{n}$ for R.A., other estimates were obtained from differences between observations during the first 2.5 years and the last 2.5 years giving $\varepsilon_{200} \leqslant 0$ ".038. Finally, clamp differences for individual stars gave $\varepsilon_{10}=0.064$. These three values of $\varepsilon_{n}$ lead to asymptotic mean errors 0 ".011 $\leqslant a \leqslant 0$ ".035 when the formula

$$
\varepsilon_{n}^{2}=a^{2}+\left(b^{2} / n\right)
$$

is applied.

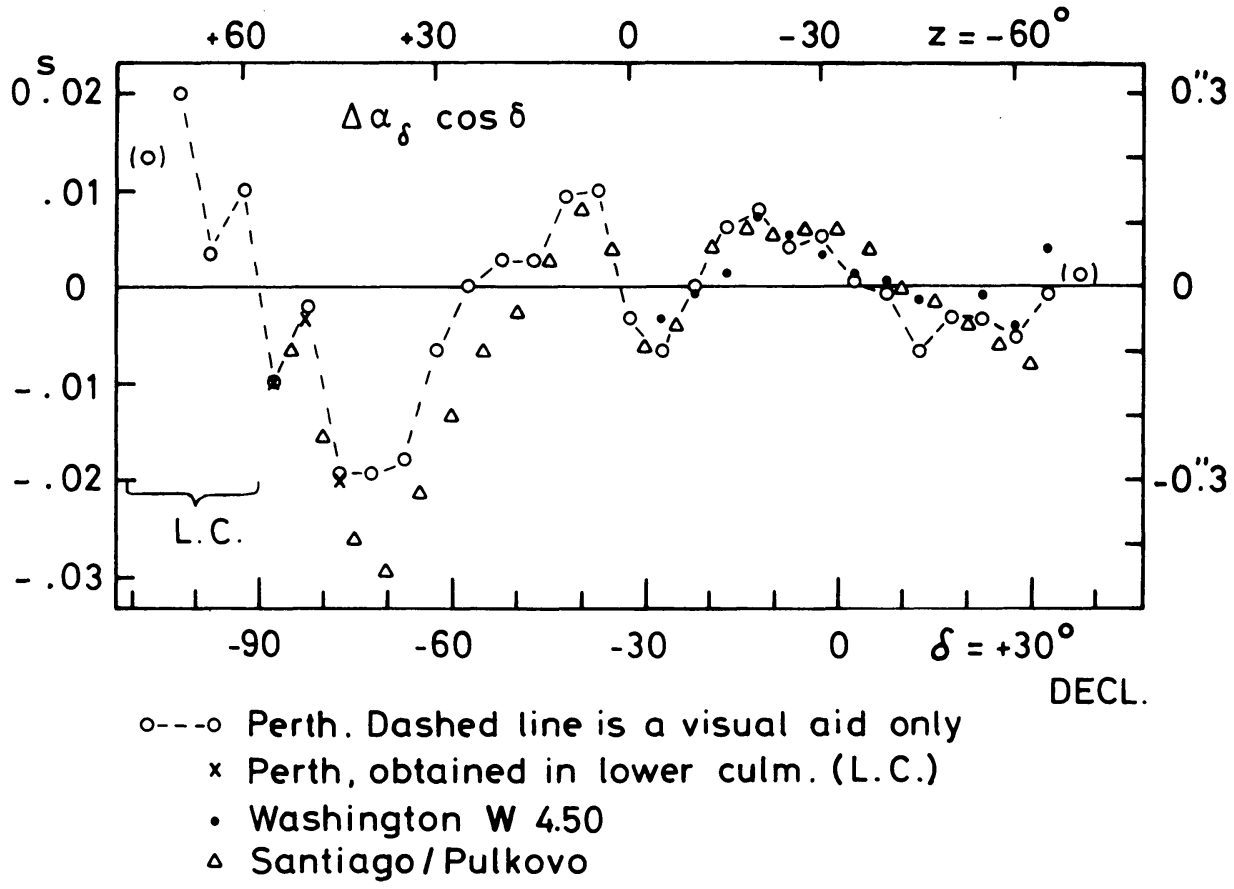

Fig. 3. Differences in right ascension from observations in Perth, Washington and Santiago in the sense (Cat-FK4).

This may be compared with the asymptotic mean error $a=0$ ".084 for R.A. in modern visual catalogues given by Kopff et al. (1964).

Figure 3 shows the values of $\Delta \alpha_{\delta} \cos \delta$ for $5^{\circ}$ zones without any smoothing between zones. Values are given for comparison from Washington (Adams and Scott, 1968) and Santiago/Pulkovo (Zverev, 1970). The values from Santiago are based on 7420 
visual observations and have been somewhat smoothed between zones by their authors. Good agreement between the three sources exists in confirming the systematic errors of FK4 notably around $\delta=-70^{\circ}$, but also in the whole interval up to $\delta=+30^{\circ}$. It is doubtful whether a new reduction of all nights with corrected positions of the FK4 stars would change our results significantly, since so many well distributed FK4 stars were used every night - on the average 43 - and in different combinations with program stars. The systematic difference of $0^{\prime \prime} .10$ between the observations from Perth and from Santiago in the interval $-75^{\circ} \leqslant \delta \leqslant-50^{\circ}$ is statistically significant, but of quite common magnitude for visual observations.

The accuracy of the Perth observations is confirmed by the observations in lower culmination which differ from the values obtained for the same stars in upper culmination by $(\mathrm{UC}-\mathrm{LC})=0.00,+0^{\prime \prime} 02$ and $+0^{\prime \prime} 01$, respectively, for the zones centered at $\delta=-87.5,-82^{\circ} .5$ and -77.5 . The zone observed at $z=75^{\circ}$, see Figure 3, does not contain all stars in FK4 and can, therefore, not be used for comparison although the accuracy at that zenith distance is still acceptable.

\section{References}

Adams, A. N. and Scott, D. K.: 1968, Publ. U.S. Naval Obs., Ser. 2 XIX, Part II.

Dejaiffe, R. J.: 1973, Astron. Astrophys. 22, 425.

Høg, E.: 1972, Astron. Astrophys. 19, 27.

Høg, E.: 1973, this volume, p. 243.

Kopff, A., Nowacki, H., Strobel, W.: 1964, Veröff. Astron. Rechen-Inst. Heidelberg, Nr. 14.

Zverev, M. S.: 1970, Izv. Glav. Astron. Obs. Pulkovo, No. 185, 3.

\section{DISCUSSION}

Fricke: I may draw the attention of the audience to the fact that the results obtained in Perth are in marvellous agreement with those obtained by the Santiago-Pulkovo team at Santiago de Chile. This strengthens very much the corrections to be applied to the FK4 system. I wish our colleagues at Perth every success for the continuation of their work. 